\title{
Perianal Endometriosis: A Rare Presentation of Extrapelvic Endometriosis
}

\author{
Kavitha D Yogini ${ }^{1}$, Devi Balasubramaniam² ${ }^{2}$ Sangeetha Karunanithi ${ }^{3}$, Ramakrishnan Parthasarathi ${ }^{4}$
}

\begin{abstract}
Endometriosis is the ectopic location of functioning endometrial glands and stroma. Perianal endometriosis can present as a painful swelling and are usual mistaken as perianal abscess, thrombosed hemorrhoids, sebaceous cyst, or a resolving hematoma. The uncommon presentation delays the diagnosis and brings agony to the patient.

Case description: A 34-year-old para 2, live 2 (P2L2), both uneventful vaginal deliveries, presented to our outpatient department with complaints of perianal pain for 3 years. Initially, the pain was more during her menstruation. Gradually it worsened and the patient started having severe noncyclical pain. There was no history of any obvious swelling or discharge from the perianal region. She gave a history of episiotomy given during her second delivery, 7 years back. There was also a history of perianal abscess drainage 2 years back. On local examination, there were no scars noted on the perineum. Per speculum and per vaginal examination were unremarkable. On per rectal examination, there were tenderness and induration noted at the 4 o'clock position. The patient was taken up for surgical exploration. The cyst wall was excised without any damage to the sphincter muscle and sent for histopathology. The histopathology report was suggestive of endometriosis.

Conclusion: Since perianal endometriosis has varying clinical presentations, this condition has to be kept in mind especially when the patient has cyclical perianal pain.

Keywords: Endometriosis, Episiotomy, Female, Painful swelling.

Journal of South Asian Federation of Obstetrics and Gynaecology (2019): 10.5005/jp-journals-10006-1659
\end{abstract}

\section{INTRODUCTION}

Endometriosis is defined as the ectopic location of functioning endometrial glands and stroma. ${ }^{1}$ Histopathologically, they are diagnosed by the presence of endometrial stroma, glands, and hemosiderin pigment, the stroma being the most essential component. ${ }^{1}$ Ovary is the most common site for endometriosis to occur. The uncommonest locations are umbilicus, laparotomy scars, hernia sacs, appendix, vagina, vulva, cervix, tubal stumps, omentum, lymph nodes, and perineum. ${ }^{1}$ The most common clinical manifestation with endometriosis is infertility, dyspareunia, dysmenorrhea, and chronic pelvic pain. Sometimes, they are asymptomatic also. The first ever perianal endometriosis was reported by Shickele in 1923. Perianal endometriosis is commonly present in episiotomy scar and, at times, found to involve the recto vaginal septum. Perianal endometriosis can present as a painful swelling and are usually mistaken as perianal abscess, thrombosed hemorrhoids, sebaceous cyst, or a resolving hematoma. This delays the diagnosis and brings agony to the patient. Cyclical pain is not a usual presentation in perianal endometriosis. ${ }^{2}$

\section{Case Description}

A 34-year-old para 2, live 2 (P2L2), both uneventful vaginal deliveries, presented to our outpatient department with complaints of perianal pain for 3 years. Initially, the pain was more during her menstruation. Gradually it worsened and the patient started having severe noncyclical pain. There was no history of any obvious swelling or discharge from the perianal region. She gave a history of episiotomy given during her second delivery, 7 years back. There is also a history of perianal abscess drainage 2 years back. She is also a diabetic on oral hypoglycemic agents $(\mathrm{OHA})$ for the past 1 year. When the patient was evaluated outside for similar complaints, MRI
1-3Department of Endogynecology, GEM Hospital, Coimbatore, Tamil Nadu, India

${ }^{4}$ Department of Surgical Gastroenterology, GEM Hospital, Coimbatore, Tamil Nadu, India

Corresponding Author: Kavitha D Yogini, Department of Endogynecology, GEM Hospital, Coimbatore, Tamil Nadu, India, Phone: +918883112537, e-mail: yoginianandvij@rediffmail.com

How to cite this article: Yogini KD, Balasubramaniam D, et al. Perianal Endometriosis: A Rare Presentation of Extrapelvic Endometriosis. J South Asian Feder Obst Gynae 2019;11(2):138-139.

Source of support: Nil

Conflict of interest: None

revealed an irregular scar tissue in the left ischio anal fossa adherent to the external anal sphincter and there was no ischio rectal abscess collection. When the patient presented to us, the general condition of the patient was good. On local examination, there were no scars noted on the perineum. Per speculum and per vaginal examination were unremarkable. On per rectal examination, there were tenderness and induration noted at the 4 o'clock position. The sphincter tone was good. Transrectal and trans perineal ultrasound (Fig. 1) revealed a perianal collection with tract extending up to the skin at the 3 o'clock position. The patient was taken up for surgical exploration. Incision on the perianal region was given from the 3 o'clock position to the 6 o'clock position and deepened (Fig. 2). About $25 \mathrm{~mL}$ of the chocolate colored fluid was drained. The cyst wall was excised without any damage to the sphincter muscle and sent for histopathology (Fig. 3). The postoperative period was uneventful and the patient was discharged on day 2 with the administration of the $\mathrm{GnRH}$ analogue. The histopathology report was suggestive of endometriosis. 


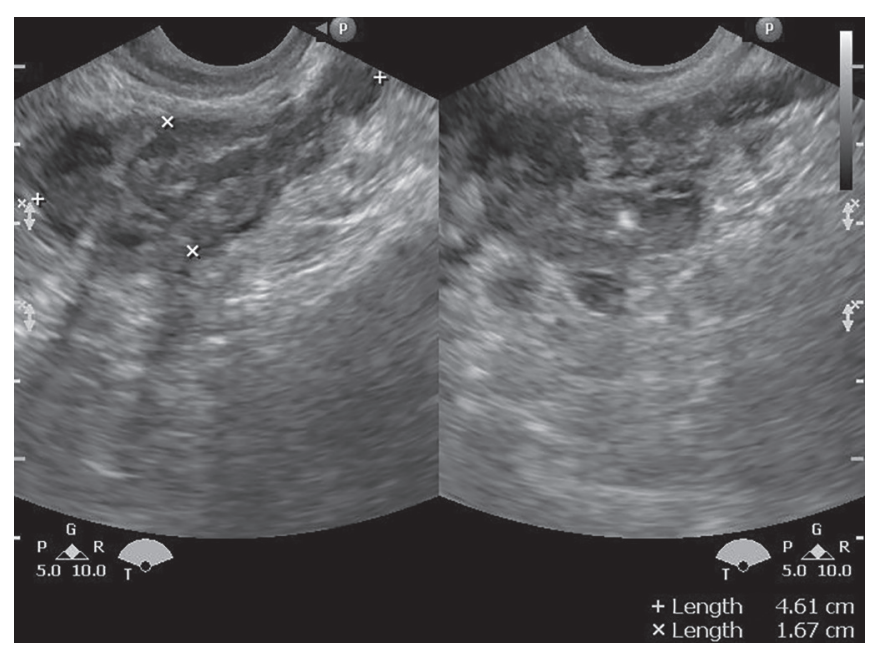

Fig. 1: Ultrasound images-transperineal and transrectal

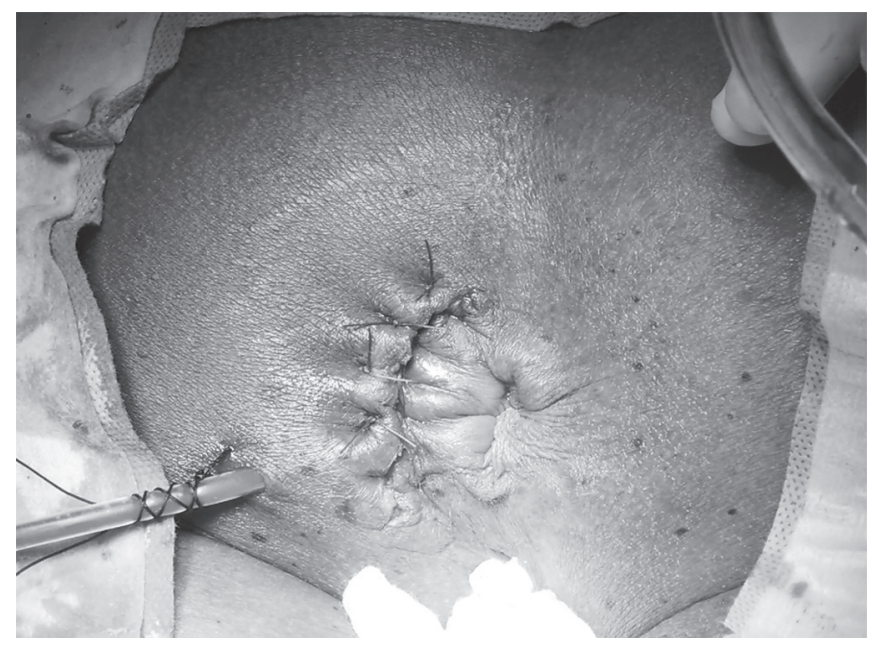

Fig. 3: Final wound closure

\section{Discussion}

Perianal endometriosis is a rarest presentation of extrapelvic endometriosis. It is seen in only $0.2 \%$ of the ectopic endometrium. ${ }^{3}$ In another study, the incidence is said to be $3.7 \%$ for perineal endometriosis. ${ }^{4}$ The diagnosis is usually delayed because of their uncommon presentation. The etiology and pathogenesis of endometriosis are still not understood clearly. Many theories have been postulated to explain its occurrence. There was no perianal swelling with cyclical pain and discharge from the swelling during menstrual cycle in our case, which made the diagnosis difficult. Ultrasound features are hypoechoic or heterogeneous mass containing cystic anechoic and hyperechoic areas. ${ }^{4}$ As the

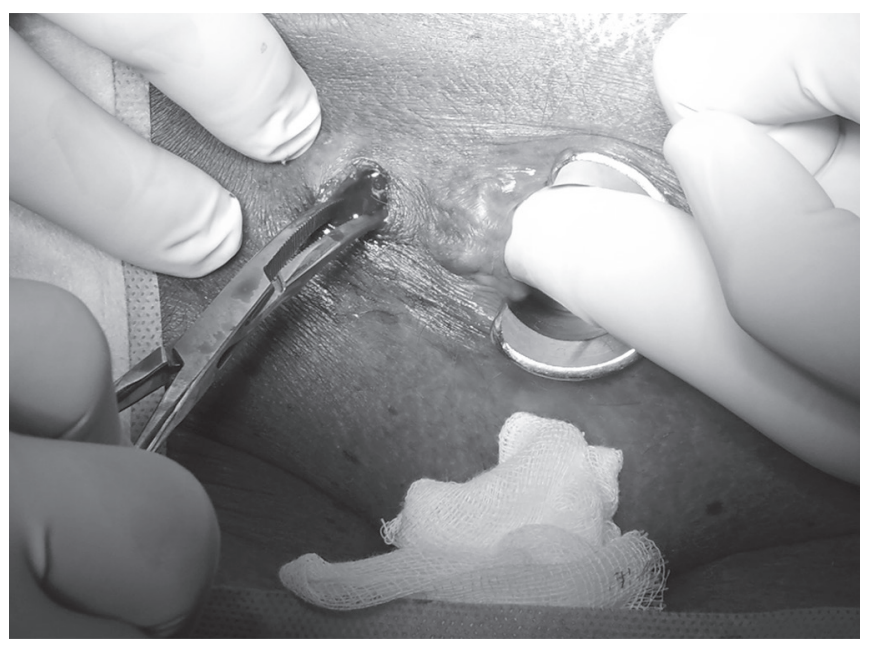

Fig. 2: Incision at the perianal region under proctoscope guidance

perineal ultrasound fail to reveal the anal sphincter involvement, an endoanal ultrasound is used in finding out the extent of the sphincter involvement. ${ }^{4}$ Pelvic MRI has greater sensitivity (90-92\%) and specificity (91-98\%) for the diagnosis of endometriomas when compared to other noninvasive methods. ${ }^{5,6} \mathrm{MRI}$ also helps in finding the extent of the anal sphincter involvement. A wide local excision with as much as preservation of the anal sphincter muscle would be the modality of treatment. When there is an involvement of the anal sphincter, preoperative hormonal therapy followed by surgical excision would be the treatment of choice. ${ }^{4}$ The recurrence rate was found to be zero when treated with both surgical and hormonal therapies. ${ }^{7}$

\section{References}

1. Gordon PH, Schottler JL, et al. Perianal endometrioma. Report of five cases. Dis Colon Rectum 1976;19:260-265. DOI: 10.1007/ BF02590916.

2. Schweiger $W, B a c h e r H$, et al. Perianal endometriosis with involvement of the external anal sphincter. Eur J Surg 1999;165:615-617. DOI: $10.1080 / 110241599750006569$.

3. Marquez J, Marquez JC, et al. Extrapelvic endometriosis involving the perineum. Rev Chil Obstet Ginecol 1995;60:1-4.

4. Zhu L, Chen N, et al. Diagnosis and Treatment of Perineal Endometriosis. In: Chaudhury K, Chakravarty B. ed. Endometriosis Basic Concepts and Current Research Trends. Hampshire: InTech; 2012. pp. 53-64.

5. Bazot M, Darai E, et al. Deep pelvic endometriosis: MR imaging for diagnosis and prediction of extension of disease. Radiology 2004;232:379-389. DOI: 10.1148/radiol.2322030762.

6. Kinkel K, Frei KA, et al. Diagnosis of endometriosis with imaging: a review. Eur Radiol 2006;16:285-298. DOI: 10.1007/s00330-005-2882-y.

7. Liang CC, Tsai CC, et al. Management of perineal endometriosis. Int J Gynecol Obstet 1996;53:261-265. DOI: 10.1016/0020-7292(95)02592-8. 